\title{
Persona y pobreza. Reflexión sobre un posible problema ético de la metodología de aprendizaje servicio
}

\author{
Javier Aranguren \\ Centro Universitario VIllanueva, España
}

\section{Resumen}

El aprendizaje servicio s una metodología de trabajo de reciente introducción en la educación universitaria europea. Por medio de él se pretende que lo que se aprende en las aulas encuentre aplicación entre personas necesitadas. Se procuran así dos fines: vivir el compromiso social de la universidad y ayudar a la comunidad (o a otras comunidades) en una situación de vulnerabilidad. Pero surgen algunas dudas éticas, pues parece evidente que se corre el peligro de usar a aquellos a los que se ayuda (a los necesitados) porque se les reduzca a un medio para vivir una experiencia de aprendizaje, conseguir unos créditos, que el profesor logre valoraciones positivas en la asignatura, etc. Sin embargo el planteamiento de la ayuda debe ser otro, pues el beneficiario es una persona, esto es, alguien con valor absoluto capaz de aportar absolutamente al que ayuda (capaz de convertirle en beneficiario). En este artículo se aboga por una aproximación benevolente, y no abstracta o siguiendo criterios de eficacia, a la metodología ApS.

\section{Palabras clave}

Aprendizaje servicio, ética, benevolencia, pobreza, voluntariado, uso. 


\title{
Individual and poverty. Reflection on a possible ethical problem of service learning methodology
}

\begin{abstract}
Service learning is a work methodology recently introduced in European university education, what is learned in the classrooms find application among people in need. Two goals are pursued: live the social commitment of the university and help the community (or other communities) in a situation of vulnerability. But some ethical doubts arise, because it seems evident that there is a danger of using those who are helped (the needy) because they are reduced to a means to live a learning experience, get some credits, that the teacher achieves positive evaluations in the subject, etc. However, the approach of the aid must be different, since the 'beneficiary' is a person, that is, someone with an absolute value who is able to contribute absolutely to the person who helps (capable of making him a beneficiary). In this article we advocate a benevolent approach, and not abstract or following criteria of effectiveness, to the ApS methodology.
\end{abstract}

\section{Keywords}

Service learning, ethics, benevolence, poverty, voluntary, use. 


\section{Una duda sobre la metodología ApS}

La metodología de aprendizaje servicio (ApS) está sin duda de actualidad ${ }^{1}$. Se trata de una herramienta con evidente atractivo, pues cumple de golpe con varios objetivos. Por un lado, relanza la vocación de servicio de la tarea (y la enseñanza) universitaria, pues el ApS pretende servir a la comunidad y en concreto a los colectivos más vulnerables de una comunidad. Por otro, apoya esa orientación hacia la práctica que ha cobrado la educación universitaria, promoviendo en los alumnos el learning by doing, pues logra situarles ante contextos reales (no simulados) de ejercicio profesional. Además resuelve la necesidad que tienen las asignaturas de grados por ofrecer créditos prácticos y actividades significativas. Por último, la vocación del ApS es la de conectar la docencia con la realidad, de modo que no son actividades de simple voluntariado (en ese caso quedarían fuera de la vida del aula), sino que se tienen que relacionar activamente con la adquisición de nuevos conocimientos.

\section{El ApS es una metodología que} comienza a levantar vuelo en Europa (en otras regiones del mundo está bastante extendida). Y como todo lo que es joven, también es una metodología que acarrea nuevos retos. Algunos tienen que ver con el excesivo número de horas de clases con que

\footnotetext{
${ }^{1}$ Sobre ApS, ver Furco (2001). Sobre su aplicación a los estudios superiores, ver Bringle y Hatcher (1996, 2000). En español, ver Aramburuzabala (2013), ver una perspectiva más crítica en Butin (2006).
}

cargan los programas, con las numerosas asignaturas, que llevan a la duda de cuánto tiempo se debería invertir en una actividad de una clase entre otras seis o diez materias. Es verdad que podría hacerse del ApS algo transversal, y proponer una actividad para varios campos de ese grado. Sin embargo las dificultades organizativas crecen en la medida en que cada vez son menos los alumnos que comparten el aula durante todo el día, pues hay muchos itinerarios entre tanto grados $y$ doble grado.

Pero los problemas no son solamente estratégicos. También pueden ser éticos. Es habitual tratar de valorar las actividades ApS desde el punto de vista de los alumnos. Incluso desde una aproximación sentimental (cómo se han sentido) que lleva a desfigurar el sentido mismo del ApS (qué hemos enseñado). Sin embargo, cabe preguntarse quiénes deben ser los beneficiarios de esas actividades: ¿los alumnos o los colectivos con necesidades? La posibilidad de reducir (al menos parcialmente) al colectivo con necesidad a un medio para que el alumno realice un aprendizaje (algo análogo a reducirlo a grupo de control con placebo para que los médicos prueben la eficacia de un medicamento) supondría su instrumentalización. Pero el punto de partida, suponemos, debe ser distinto: mantener siempre despierta la conciencia de que el otro, el débil en este caso, es un ser humano, con una dignidad absoluta, del que en ningún caso vale servirse para un fin instrumental. "Obra de tal modo que uses a la humanidad, tanto en tu aprendizaje servicio. RIDAS, Revista Iberoamericana de Aprendizaje Servicio, 5, 110-122. 
persona como en la persona de cualquier otro, siempre al mismo tiempo como fin y nunca simplemente como medio" (Kant, 2006:429).

Con el ApS puede ocurrir como con la lucha contra la pobreza: que impere una racionalidad de lo abstracto que acabe cegada para el encuentro con la persona. Nuestra reflexión va a ser filosófica, no empírica o positiva, con una pretensión más de llamada de atención y advertencia que de denuncia moral.

\section{La pobreza, los pobres, esta persona}

La ciencia positiva cifra su avance en la cuantificación. Muchos saberes buscan el sobrenombre de ciencia, con lo que esta conlleva de objetividad, universalidad, verificación, prestigio. Hasta conocimientos que tenían que ver más con lo poético (como la historia, en su dimensión de estudio de las acciones particulares) o con lo prudencial (como la educación, y todas las artes de lo que tiene que ver con las decisiones libres de los sujetos de las virtudes) suelen preferir, en cuanto entran en el terreno de la literatura académica, criterios empíricos, medibles, que se puedan presentar en forma de tablas. Lo abstracto permite hacer ciencia, lo concreto resulta demasiado particular, y solo cabe contarlo: la narración, la poética.

La pobreza pertenece a lo cuantitativo. Sus números producen vértigo. En 2012, informaba el Banco Mundial, 896 millones de personas subsistían con menos de 1,90 dólares por día. Y 2.200 millones lo hacían con menos de 3,10 dólares (menos de $3 €$; menos de $90 €$ al mes por persona). Europa y USA sumados cuentan con menos habitantes que los más pobres entre los más pobres ${ }^{2}$. Imaginemos ahora el $100 \%$ de Europa y USA viviendo con menos de $3 €$ al día. A cada uno de nosotros mismos. Si se consigue contemplar esa imagen (con sus consecuencias: ausencia de trabajo, falta de ropa, penoso nivel de vivienda, carencia de higiene, hambre, ausencia de servicios públicos -médicos, colegios para los niños-, falta de posibilidad de cambio), podremos empezar a hacernos una idea de qué significa no solo ser pobre, sino que yo soy pobre.

Un pobre no ahorra nada, vive arrojado en el presente, queda fuera de la vida civil pues al estar todo el día ocupados en sobrevivir ahora no tienen tiempo de hacer oír su voz (que a menudo se encuentra secuestrada en manos de los poderosos). Su vida en el puro presente les arroja fuera de la Historia (History, no tienen ni pasado ni futuro) y de la historia (story, no tienen nada que contar). Se trata de gente excluida de la sociedad, a los que se niega la palabra, y se les amenaza con violencia e inseguridad ${ }^{3}$.

¿Bastaría con argumentar cosas como la siguiente? De una población de 43 millones, solo 7 viven en chabolas urbanas. iNo puedes hablar de pobreza

\footnotetext{
${ }^{2}$ Cf.http://www.bancomundial.org/es/topic/pove rty/overview.

${ }^{3}$ Amnesty International, Kenya: The unseen majority. Nairobi's two million slum-dwellers, 2009,https://www.amnesty.nl/sites/default/files /public/rap_kenia_the_unseen_majority.pdf.
} aprendizaje servicio. RIDAS, Revista Iberoamericana de Aprendizaje Servicio, 5, 110-122. 
real en este país!. Es un comentario al autor de este artículo hecho por un importante empresario keniano. $Y$ es verdad: desde el punto de vista de los indicadores macroeconómicos no son tantos los necesitados: isolo el 33\%! Además, se puede casi llegar a vivir sin verlos.

Hay muchos programas enfocados al desarrollo. Hay espacio para ideas magníficas, como el uso de dinero a través del teléfono o de la energía solar pagada en plazos de céntimos diarios. El Banco Mundial, el World Food Program, ONU, Cruz Roja, UNICEF... hacen muchas cosas. Incluso cada vez más universidades de países del primer mundo entienden la conveniencia de promover lo solidario: porque los alumnos lo reclaman, porque es una herramienta de marketing, porque ahora no se entiende una gran empresa que no se preocupe por el papel libre de cloro y por este otro tipo de temas. Incluso porque les parece realmente importante, y cada vez que unos alumnos o un profesor han hecho algo en este sentido (bajo forma de voluntariado, bajo la forma de ApS) la comunidad universitaria se ha visto reforzada en su identidad y en su misión. Y eso lo muestran las encuestas que se pasan al final de cada asignatura: los alumnos que hacen el bien valoran hacerlo.

Sin embargo, ¿ha superado este tipo de ayuda el nivel de la abstracción? Luchar contra la pobreza puede ser como luchar contra el enemigo en la guerra. Es algo que puede vivirse en el olvido de la identidad del otro ${ }^{4}$. Así, la lucha contra la pobreza se puede centrar en los números, reduciendo las personas a individuos, a casos de un universal. Pero el método aritmético resulta inadecuado para un ser en el que lo más propio del individuo es que no es individuo (un caso singular de un universal, como un tornillo), sino un ser personal. Ante el hombre "el bien colectivo no justifica los medios" (Finkielkraut, 1998, 79.), ni se puede subordinar el individuo a la colectividad.

Se puede afirmar: iHacemos muchas cosas para aliviar la pobreza!. Se puede añadir: iNuestro plan de estudios cree que nuestros alumnos deben añadir a su currículo la dimensión solidaria! y, al mismo tiempo, ser un perfecto extraño a los problemas de los pobres. Heidegger (2001,221), en Ser y Tiempo, desarrolla la cuestión de "la cotidianidad del ahí y la caída del Dasein". En su descripción de los radicales de la vida humana (la cháchara, la curiosidad, la ambigüedad) subraya lo costoso que le resulta al hombre superar la capa más externa de la realidad: cómo los seres humanos tenemos serios problemas para ir más allá de los lugares comunes, para ser responsables, para centrarnos en el cuidado. Vivir en la abstracción (en la apariencia platónica)

\footnotetext{
${ }^{4}$ Hay otro debate, en parte relacionado con nuestro modo de acercarnos a la pobreza: la discusión entre las grandes soluciones, las respuestas macro de las grandes organizaciones y la ineficacia estructural de las mismas (Sacks, 2005) y las respuestas contra él (Moyo, 2009; Easterly, 2006).
} aprendizaje servicio. RIDAS, Revista Iberoamericana de Aprendizaje Servicio, 5, 110-122. DOI10.1344/RIDAS2018.5.8 
es la normalidad no natural del ser humano.Y decimos no natural porque estamos hechos para lo realmente real, aunque casi nunca lo alcancemos.

Heidegger (2001) describe como inautenticidad aquella situación del que se encuentra totalmente fascinado por el mundo y por la existencia con los demás en el se (lo impersonal, el se dice, se piensa). Esa presencia impersonal se acepta, se asume, de forma natural y acrítica. Uno se puede sentir bien, hace fotos y se hace fotos con monumentos de fondo, con niños de otras razas sonrientes de fondo, valorando la sensación subjetiva que dan las cosas bien hechas, inconsciente del ser de quien tiene delante, atento tan solo a la imagen que esas caras proyectan en los nuevos areópagos digitales y a finalizar otra de las prácticas a las que había que añadir el tick, la marca, de la actividad realizada, evaluada, aprobada, y que abre al estudiante hacia nuevas metas de su proceso de aprendizaje.

\section{Aprender a mirar al otro:hospitalidad}

¿Cómo evitar que una actividad ApS termine en abstracción? ¿Cómo dejar de valorar sobre todo la dimensión sentimental - el cómo me he sentido, el cómo me ha llenado- de esa actividad? ¿Cómo hacer ver que lo accidental de una acción relacionada con la solidaridad -desde el voluntariado al ApS- es justamente el estudiante y las reacciones anímicas del estudiante, y que lo esencial es la persona que recibe ese servicio? ¿Cómo entender que la comprensión contraria es abiertamente inmoral -el pobre como un medio para que el alumno mejore como persona, para que el joven del primer mundo se dé cuenta de su suerte y disfrute más de lo que tiene, para que tal vez alguno decida dedicarse a la cooperación en el futuro-?

Descubriendo el ser personal. La persona no se relaciona con la cantidad. Es un nombre propio al tiempo que común, como la palabra yo es una palabra que decimos todos, pero que cuando la uso yo indica mi condición de absoluto. Cada persona es solo ella misma: una matanza no es mala tanto por el número, como por cada una de las víctimas. El bebé de tres meses que murió en la bomba de Nagasaki es tan importante como el hombre que descubrió la penicilina. Hay algo en el ser humano (la fuente más radical de su ser, la persona) que va más allá de la naturaleza, de lo universal, de la generalidad, de la definición, de la idea. Cada persona es un absoluto; alguien, no algo, que tiene tal valor en sí mismo como el grupo de todos los demás. Salvar a un hombre es lo mismo que salvar a toda la humanidad. Usar a un hombre es lo mismo que despreciar la dignidad de toda la humanidad. Y somos capaces de realizar estos dos tipos de acciones.

¿Cómo se alcanza esa experiencia de lo absoluto? Usamos la palabra experiencia porque a la persona no se llega como conclusión de una cadena de silogismos. La persona no es un concepto: no es un qué, sino un quién. La experiencia consiste en el encuentro. Lo absoluto aparece en la experiencia

Aranguren, J. (2018). Persona y pobreza. Reflexión sobre un posible problema ético de la metodología de aprendizaje servicio. RIDAS, Revista Iberoamericana de Aprendizaje Servicio, 5, 110-122. DOI10.1344/RIDAS2018.5.8 
de la mirada que se cruza con la mirada. Primero se abren los ojos, se despierta de la oscuridad (de la apariencia, del impersonal se, de lo generalizante), se despierta a la realidad. Dicha experiencia que no ocurre con las cosas, con el dinero. Tampoco con los animales, que no pueden ser amigos porque en ellos no hay reciprocidad, no son capaces de mirar de vuelta, en palabras de Santo Tomás de Aquino. Esa experiencia solo puede darse con otra persona, ante la que cabe un encuentro que escapa al propio control, a los prejuicios y previsiones que uno tuviera.

Levinas (1979) llama a esta experiencia hospitalidad. En ella se presupone la no adecuación del otro: no se le puede reducir a nuestras categorías. Dicho en relación con la pobreza: el pobre no es el pobre. Es un sujeto, un ser personal, un misterio, una infinidad de posibilidades por conocer. No es alguien que se reduzca a una categoría (ser pobre). No es alguien que se encuentre esperándonos para ser. Si el otro es recibido desde la hospitalidad simplemente se le da cobijo en la mirada, en la mano, en el abrazo, en la sonrisa.

La hospitalidad significa que se reconoce al otro en su ser, y no como alguien que pueda añadir algo a mi ser (placer, utilidad, interés). El amor de dar, esa hospitalidad, el amor de benevolencia, resulta mucho más elevado que cualquier amor de necesidad, que la actividad que se hace "porque reporta un bien a la comunidad, a nuestros alumnos, a nuestra vocación solidaria, a nuestra imagen de marca, a nuestra nueva idea pedagógica, a nuestro estar siempre con lo último y desechar lo anterior, a una actividad que reporta muchas valoraciones favorables" (Levinas, 1979: 27).

La hospitalidad, el amor de benevolencia, es una relación en la que no se busca (ni siquiera colateralmente) el beneficio. En ella el otro es visto como otro (la fuente originaria de una nueva visión del mundo, tan nueva como puede ser la propia, incluso en su en su condición de niño o de enfermo, en su pobreza). Y ante él reacciono como alguien que ha visto la necesidad de cuidar, de ayudar, si hubiera obstáculos que impidieran el desarrollo de la intimidad de esa persona, o la expresión de esa intimidad por medio de las obras que debería ser capaz de llevar a cabo.

"Romper el marco de un contenido pensado, cruzar las barreras de la inmanencia... La idea de infinito (que no es una representación del infinito) es sujeto de actividades" (Levinas, 1979:27) y por eso no le basta con ser dicha: tiene que ser vivida. La persona se experimenta en la co-existencia, en la relación. No hay marcos, no caben generalizaciones: hay que tratar con ella, escucharla, aceptarla. La gran organización llevará sacos de trigo, programas de desarrollo, pero no agarrará la mano ni mirará a los ojos. Cuando pase el hambre, el beneficiario podrá pensar: ¿Piensan sobre mí, o solo soy un número? ¿Les importo? ¿Les daría lo mismo si en vez de mí fuera otro?. Todas estas preguntas, para quien se las hace, resultan aprendizaje servicio. RIDAS, Revista Iberoamericana de Aprendizaje Servicio, 5, 110-122. 
desasosegantes. Y acaban convirtiendo la ayuda en una carga bajo el nombre de paternalismo. O acaban por no respetar la iniciativa de los beneficiarios. Falta iniciativa de parte de estos a veces porque conscientemente se evita, y se les pide que no trabajen pues ya se les provee de comida; a veces porque se les ha deseducado, han caído en la dependencia, y no se han generado ni los recursos ni la ilusión que se necesitan para salir de ella; a veces porque lo que reciben hunde la pobre economía local, pero ningún donante se ha planteado esa posibilidad.

El paternalismo es la gran tentación de la cooperación. Tratarles como si fueran niños incapaces de cuidarse a sí mismos. No ponerse en su piel; querer conquistarles el bienestar antes de atender a su experiencia del mundo o de comprender sus costumbres; no reconocer su identidad y no dejar que reconozcan la identidad del que ayuda (que actúa como cooperante, como voluntario, y no como sí mismo...).

De todos modos se ha aprendido mucho sobre esto, gracias a los errores y la experiencia. En cambio, esa historia no necesariamente existe ni en el voluntariado ni en el ApS, y la posibilidad de causar daño aumenta precisamente por esta carencia de expertise. $Y$ no basta con consolarse con un por lo menos los daños no serán muy grandes, pues somos pequeños pues, como se ha dicho, entre los seres personales cada uno vale exactamente lo que todo el resto: todo.

\section{Benevolencia: la verdadera}

\section{experiencia del otro}

Benevolencia 5 . Por ella tomamos cuidado de los intereses del otro. Por ella vemos las cosas según su fin, no por su función. Por ella el otro deviene real para mí. Y eso ocurre sobre todo en el acto de amar, siempre que amemos deseando el bien del amado ${ }^{6}$. Por la benevolencia uno llega a entender que las cosas no tienen valor porque se relacionen con nosotros. La razón de ayudar no puede ser la evaluación, o el aprendizaje, o sentirse a gusto: es el bien del necesitado, en la medida en que lo necesita y en que la ayuda se corresponde con sus necesidades (ino con las nuestras!). La benevolencia es la intuición de estar en el mundo para amar las cosas y a las personas como merecen ser amadas, y de tener la responsabilidad de ayudarles a cumplir con sus fines.

Nuestra mirada debe superar las capas de la exterioridad, la apariencia, y llegar hasta el hecho de que el otro mi pobre, el negrito- es una subjetividad: "Lo que dice Aristóteles de la substancia puede ser predicado de la subjetividad. Todas las demás cosas se dicen de ella, pero ella no se dice de ninguna. No es una característica de un ser. En vez de eso, simplemente es. Y precisamente es una imagen en este ser simple; es el absoluto en la forma de una imagen" (Spaemann, 2000: 95-96) porque ese sujeto es el que es de un modo completamente nuevo respecto a las demás cosas: cada persona es una

\footnotetext{
${ }^{5}$ Para muchas de estas ideas, a Spaemann, R. (2000), 92-105.

${ }^{6}$ Suma Telógica II-II, q. 23, a.1.
} aprendizaje servicio. RIDAS, Revista Iberoamericana de Aprendizaje Servicio, 5, 110-122. DOI10.1344/RIDAS2018.5.8 
novedad para el universo.

¿Por qué no se puede matar? Esta expresión no señala una imposibilidad fáctica: matar es fácil, y de hecho se trata de una acción estadísticamente muy frecuente. Es una prohibición moral que nace de la experiencia benevolente del otro. Esta experiencia se produce en la epifanía del rostro del otro, epifanía que es ética (Levinas, 1979). El miedo a matar a alguien nace de la santidad o carácter sacro de la realidad del otro. Es lo santo que experimento en mí lo que me hace capaz de reconocer en el otro a mi igual (iNo debo ser tratado nunca solamente como medio!). No ser capaz de reconocer esa dimensión en los demás no es culpa del otro, sino de la propia ceguera del sujeto que no es capax rei, capaz de realidad, sino que se ha quedado enfangado en la banalidad burocrática de lo abstracto.

Si cambiamos la figura del asesinado por el pobre, y la del asesino por el voluntario, el alumno de ApS o el diseñador de la actividad, ¿qué queda? Queda la posibilidad de cambiar el lugar común. Sería bueno sensibilizar a nuestros alumnos; El ApS es una metodología que se está usando en muchas universidades de prestigio; ApS es un asunto fantástico para investigar porque todas las revistas de innovación pedagógica están publicando sobre él, del mismo modo en que hace diez años lo hacían sobre las pizarras electrónicas; El 37\% de los alumnos que han hecho tres o más actividades ApS dedican un 6\% o más de su tiempo profesional a actividades pro bono.
Todas esas afirmaciones son externas al significado de la ayuda. ¿Dónde están los pobres?, ¿Qué ha sido de ellos? ¿Les dejamos entrar en nuestra intimidad? ¿Dejamos que nos incomoden, que remuevan las estructuras de nuestras seguridades? Si se acerca un niño pidiendo dinero, ¿Somos capaces de pensar que él preferiría estar jugando? ¿Vemos en él a los propios hijos, a los hijos de los amigos, a los hermanos pequeños? ¿Nos ponemos en su situación? ¿Nos damos cuenta de que el niño no ha elegido el lugar en el que se encuentra? ¿De que sus padres tampoco, aunque anden ahí metidos desde siempre? ¿De que nunca han podido pensar en formarse o en empezar un negocio porque viven en el ahora, porque tienen que lograr sobrevivir? ¿Lo vemos, o diseñamos una actividad ApS, hacemos una actividad ApS, nos sentimos algo mejor y conservamos durante un breve tiempo algunas fotos de recuerdo de tantas y tan pasajeras emociones?

¿Quién elige vivir en la pobreza? ¿Quién elige limitarse a sobrevivir? Nuestra imaginación, ese conocimiento superficial por el cual catalogamos la realidad para hacerla simple y sistematizada, nos mueve a pensar que su situación es en el fondo el resultado de una elección. La perspectiva benevolente, en cambio, invita a ponerse en la piel del otro: Cómo sería mi vida si... Y podemos Ilenar con facilidad los puntos suspensivos: ... mis hijos tuvieran que mendigar; ... mi salario estuviera por debajo de los dos euros diarios; ... si no tuviéramos ni una letrina en casa ni dinero para 
pagar un baño público; ... si la dieta de mis hijos fuera siempre pobre en nutrientes; ... si no fuera capaz de pagar por su educación y les estuviera así perpetuando en la pobreza. Y lo que se quiera imaginar.

¿Y cómo me gustaría que fuera la reacción de quien viniera a encontrarse conmigo? ¿Curiosidad? ¿Rechazo? ¿Afán por hacerme fotos a mí o a mis hijos sin consultármelo, sin estar muy seguro de querer ser un animal de feria en la feria de las caridades? ¿Cálculo por su parte para ver qué se puede sacar de ese encuentro en forma de experiencia, prestigio, currículo, nota, valoración por parte de los alumnos, estar en la cresta de la ola de las corrientes pedagógicas? ¿No nos bastaría con una reacción de bienvenida, de coresponsabilidad, de igualdad, de respeto? Eso - que el que viene a mi encuentro me viera como a un igual y por lo tanto dejara espacio para mi palabra sin imponerme ni su proyecto ni sus presupuestos - sería benevolencia.

\section{Despertar a la realidad y la cuestión de los números}

"El otro es importante para mí por lo que en sí mismo es, no por lo que sea para mí. Tras esta paradoja se esconde lo que hemos descrito como despertar a la realidad. (...) Solo en esta completa realización el otro se nos convierte en real pues, aunque a menudo nos parece que ellos simplemente están allí, no son para nosotros lo que son en sí mismos. Solo podemos entender lo que significa ser un yo porque pasamos por el hecho de que nosotros somos un yo. $Y$ por eso, aunque tenemos instinto, también somos capaces de trascender nuestra centralidad y percibirnos como el otro del otro, y al otro, por su parte, como alter ego" (Speamann, 2000: 98), como otro yo.

Spaemann (2000: 194) también afirma que "el paradigma de acción benevolente es cualquier acción con la que acudamos en socorro de la vida humana necesitada de ayuda". Las llamadas obras de misericordia hablan de esta evidencia. Pero el socorro no es la sustitución. Ni tampoco socorrer es mediatizar: el otro, como fuente de fines, siempre puede ser también para mí fuente de ayuda. Lo que necesito es mirarle a los ojos, tratarle de igual a igual, vivir desde el desinterés la intención de apoyarle en lo que necesite, no en lo que yo quiera que necesite, no en lo que yo quiera que quiera.

El amor de benevolencia deja ser al amado, a la vez que lleva a la responsabilidad de sostenerle en la consecución de su fin último (la perfección, la vida buena), el cual no está asegurado y en determinadas circunstancias (por ejemplo, la pobreza extrema, económica o moral) se hace objetivamente difícil.

Si cada persona es un fin, a la hora de plantear la ayuda, los criterios de eficacia o productividad no son los más válidos: cada uno que mejore habrá merecido la pena. ¿Es mejor llegar a 10 que a uno? No hay esfuerzo grande, no hay esfuerzo pequeño: la racionalidad económica hace cálculos de gasto en las acciones médicas; los padres de un

Aranguren, J. (2018). Persona y pobreza. Reflexión sobre un posible problema ético de la metodología de aprendizaje servicio. RIDAS, Revista Iberoamericana de Aprendizaje Servicio, 5, 110-122. DOI10.1344/RIDAS2018.5.8 
niño enfermo, no. Ellos estarían dispuestos a dejarse la fortuna, a captar fondos, a hacer lo imposible, por curar tan solo a su hijo: él lo vale todo. Ellos tienen un amor benevolente. El ministerio de salud juega con la abstracción de los grandes números, su amor es abstracto.

Del mismo modo pasa con la ayuda: ¿en qué debemos invertir: en llevar a niños al colegio, en gastos médicos, en comida para hoy, en sonrisas y compañía? ¿Con qué criterio debemos elegir a los beneficiarios? ¿Su coeficiente intelectual? ¿Nos centramos en los más inteligentes? ¿Qué criterio?, ¿el que si sanan tengan posibilidad de hacer un trabajo útil? ¿Dejamos un poco de lado a los enfermos incurables, o a los ancianos? ¿O más bien el criterio debe ser la falta de criterio, sean quienes sean, sin importar quiénes sean?

Cada ser humano, cada persona (el listo, las personas con diversidad funcional intelectual, la mujer o la niña, el que tiene por padre a un alcohólico maltratador, el deprimido), vale lo que todo el universo. Eso, que sabemos de nosotros mismos, lo podemos proyectar en el otro. $Y$ eso destroza por completo la primacía de la racionalidad económica (o de la eficacia educativa) en las actividades ApS, si es que estas quieren dar cuenta de la verdad del hombre, y si quieren que el protagonista sea no la actividad, sino la persona que recibe (que comparte con el donante) el apoyo o la ayuda.

No puedo resolver el problema de la pobreza. ¿Quién puede? Pero en cambio si puedes colaborar con este hombre, poneros hombro con hombro (sin hacerle dependiente, sin aprovecharte de él para educar, aprender o sentirte mejor) y mejorar en algo su vida.

¿Pero cómo se logra esa rectitud en la que el que ayuda no se aprovecha para educar, aprender o sentirse mejor? Sería un error considerar que sea necesario renunciar a todas estas cosas. Lo que es importante es que no sean nunca la motivación principal. Lo dice Aristóteles hablando de la amistad: no hay amistad mejor que la perfecta (la amistad benevolente, en la que se cuida del amigo por el amigo mismo, en la que se le quiere porque sí), que a la vez es la más placentera y la más útil. Si con la ApS se quiere enseñar, es fundamental que la orientación de la actividad y la sensibilidad de los alumnos sea la de donación total. De otro modo no solo se falseará la actividad, sino que perderá su eficacia, se meterá - como tantas veces el amor y la amistad reales- en el ámbito del cálculo y del provecho propio.

El amor es una relación entre tú y yo. Dos miradas en reciprocidad. No trata de los grandes números. La amistad es algo exclusivo, abierto solo a unos pocos. Ocurre lo mismo con la familia y con el amor matrimonial. Este es el modo real del amor del ser humano: uno a uno (incluso cinco veces uno, para una madre de cinco niños). Somos finitos. No somos Dios: abarcamos únicamente un número limitado de problemas y de gente. Sin embargo, como ocurre con la virtud, a fuerza del

Aranguren, J. (2018). Persona y pobreza. Reflexión sobre un posible problema ético de la metodología de aprendizaje servicio. RIDAS, Revista Iberoamericana de Aprendizaje Servicio, 5, 110-122. 
ejercicio del amor el corazón puede ensancharse y llegar a más y más. Y se ensancha en la medida en que aprende a aprender del otro, y queda convencido de que la mejora y el enriquecimiento tiene doble dirección. El ApS no debe buscar imponer un modo adecuado de entender la vida, sino que debe servir de punto de encuentro entre dos realidades, y ser un encuentro en que esas dos realidades se mejoran entre sí, aprenden la una de la otra.

¿No podríamos decir que solo haremos auténtico ApS si somos capaces de ver, y de hacer ver a nuestros alumnos, la riqueza que pueden descubrir en la pobreza? ¿Si somos capaces de ver que no están ayudando sino compartiendo dos realidades - la suya, la de el Otroque tienen que ser complementarias? Así el otro no es un beneficiario (categoría demasiado abstracta) sino un compañero, un igual, un hermano.

Así se cumpliría con creces la definición que hacía sobre el ApS Robert Sigmon (1979), un acercamiento a la educación por medio de la experiencia que se basa en la premisa del aprendizaje recíproco.

\section{Referencias bibliográficas}

Amnesty International (2009). Kenya: The unseen majority. Nairobi's two million slum-dwellers, https://www.amnesty.nl/sites/default/fi les/public/rap_kenia_the_unseen_majo rity.pdf.

Aramburuzabala, P. (2013).

Aprendizaje-servicio: una herramienta para educar desde y para la justicia social. Revista Internacional de Educación para la justicia social, 2(2), 5-11.

Astin, A. W.; Vogelgesang, L. J.; Ikeda, E. K.; y Yee, Jennifer A. (2000). How Service-Learning Affects Students, Higher Education. Disponible en https://digitalcommons.unomaha.edu/s Icehighered/144

Bringle, R. G. y Hatcher, J. A. (1996). Implementing Service-Learning in Higher Education, The Journal of Higher Education, 67(2), 221-239.

Bringle, R. G. y Hatcher, J. A. (2000). Institutionalization of Service-Learning in Higher Education, The Journal of Higher Education, 71(3), 273-290.

Butin, D. W. (2006). The Limits of Service-Learning in Higher Education, The Review of Higher Education, 29(4), 473-498.

Easterly, W. (2006). The White Man's Burden. Why the West Effort to Aid the Rest Have Done so much IIl and so little Good. New York: The Penguin Press.

Finkielkraut, A. (1998). La humanidad perdida. Ensayo sobre el siglo XX. Barcelona: Anagrama.

Furco, A. (2001). Service Learning: The Essence of the Pedagogy. Conneticut: Information Age Publishing.

Furco, A. (1996). Service-Learning: A Balanced Approach to Experiential Education, Service Learning, General, 128. aprendizaje servicio. RIDAS, Revista Iberoamericana de Aprendizaje Servicio, 5, 110-122. 
Heidegger, M. (2001). Being and Time. London: Blackwell.

Kant, I. (2006). Fundamentación de la Metafísica de las Costumbres. Madrid:

Tecnos.

Levinas, E. (1979). Totality and

Infinity. London: Martinus Nijhoff Publisshers.

Moyo, D. (2009). Dead Aid. Why Aid is not Working and How there is Another Way for Africa. New York: The Penguin Books.

Sacks, M. (2005). The End of Poverty. New York: The Penguin Press.

Sigmon, R. L. (1979). Service-learning: Three Principles, Synergist. National Center for Service-Learning, ACTION, 8 (1), 9-11.

Spaemann, R. (2000). Happiness and Benevolence. Paris: University of Notre Dame Press. aprendizaje servicio. RIDAS, Revista Iberoamericana de Aprendizaje Servicio, 5, 110-122. 